\title{
Understanding the design variables that contribute to the response of a prosthetic foot: Part II - Rig Validation
}

\author{
James Hawkins \\ Department of Design and Engineering \\ Bournemouth University \\ Poole, BH12 5BB, UK \\ james@ridebo.com
}

\author{
Philip Sewell, Mihai Dupac, \\ Department of Design and Engineering \\ Bournemouth University \\ Poole, BH12 5BB, UK \\ psewell@bournemouth.ac.uk
}

\begin{abstract}
In this paper the validation of a rig capable of simulating the dynamic response of an energy storing and returning composite prosthetic foot during amputee running is considered. It is shown that the rig can simulate the running action of an amputee runner and return similar ground contact time. It has also been shown that if a small input force is applied in a timed and sympathetic manner to the prosthetic spring-mass system the amplitude of oscillation increases which agrees with results of previous research. The rig will allow the design variables that contribute to the response of a prosthetic foot during running to be understood and optimised to possible improve the foot's performance.
\end{abstract}

Keywords - prosthetic; foot; displacement; stiffness; running; amputee; boundary conditions; design; optimisation

\section{INTRODUCTION}

Numerous research projects have focused on the development of adaptive and active foot designs [1, 2, 3, 4]. Recent published work $[5,6,7]$ has demonstrated the link that exists between impulse timing and the energy return from a prosthetic foot. Along with the mentioned research, inconsistency in measurement techniques and obtained results in comparing various feet have been observed. Geil [8] focused on the hysteresis and efficiency of energy return of a variety or ESR (Energy Storing and Returning) feet using a dynamic hydraulic testing machine using Two Teflon sheets placed between the table and the foot to minimize friction during foot loading and deformation. The resulting hysteresis loops are therefore more likely to have occurred as a result of the friction in the slippage system under load than from the damping properties of the spring itself.

Other investigations have taken place using similar techniques but none have exactly replicated this same methodology. Repeating such an investigation in an accurate manner would be impractical given that the friction coefficient $(\mu)$ between the toe and test machine would need to be precisely replicated. Buckley [9] summarises that 'The findings in the present study, indicate that $100 \%$ of the energy absorbed by the (Sprint-Flex or Cheetah) prosthesis was returned'; a direct contradiction of the work carried out by [8] who defined the efficiency of a Flex Foot prosthesis as 75\%. Czerniecki
[10] proposes a value of $84 \%$ for the same model of foot whereas [11] suggest: 'The material behaviour of the carbon keels of the dedicated prosthesis provided a hysteresis of less than 10 per cent, indicating a high per cent of energy return'. All of the measurement approaches mentioned previously concern the isolation of a prosthetic device and subsequent analysis using laboratory equipment. However numerous studies are available that observe human running and record specific variables. The technique chosen should reflect the parameters demanded by the research.

Previously authors $[12,13,14,15]$ have suggested the design of a dynamic response test fixture that aligns with the assumption of a spring-mass system (Fig. 1). Using this approach the validation of a rig effectively capable of replicating the dynamic response of a prosthetic foot during amputee running is considered. This allows the variables that contribute to the response of a prosthetic foot to be manipulated and their effect understood to establish parameters that would lead to a better performing foot. For example, if a small input force is applied in a timed manner to the prosthetic rig the amplitude of oscillation will be expected to increase. Furthermore the ground contact point could change depending on running velocity, foot deflection, stride length, stride frequency or even the individual set up of the prosthetic foot.

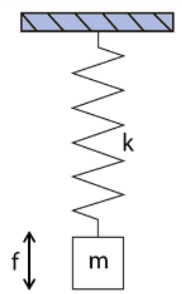

Fig. 1. Ideal spring-mass system where $\mathrm{f}=$ frequency $(\mathrm{Hz})$ of oscillation, $\mathrm{k}=$ spring stiffness $(\mathrm{N} / \mathrm{m})$ and $\mathrm{m}=$ mass $(\mathrm{kg})$

\section{RIG VALIDATION}

\section{A. Comparison with Amputee Running}

Before investigative work can be conducted the validity of the rig as an alternative to amputee running should be 
evaluated. The reason for fabricating the rig is to allow the controlled testing of feet without the need for using an amputee. Variables (such as the mass applied, deflection amplitude and ground contact points) can be modified in a repeatable and iterative manner and the response of the foot can be examined for future design optimisation. Using amputees for comparative analysis has caused problems for researchers in the past and it is the intention of this investigation to avoid a repeat of such issues. However the rig makes some assumptions and the precise action is clearly different from a real-world environment in which no two steps are the same.

The most effective and robust manner of validating the rig is to attempt to replicate the action of the amputee runner, which in return will give a direct comparison between the runner and the rig and allow conclusions to be drawn as to the effectiveness and suitability of further study using the rig.

The data was collected from a long-term and regular user of an ESR prosthetic foot who did not suffer from extreme or influential pathologies such as restricted movement or chronic pain that might adversely affect running style or repeatability. The participant was a 32 year old male left-side uni-lateral trans-tibial amputee who had been using a prosthesis for over ten years following a trauma. The participant had been the user of a category $6 \mathrm{Hi}$ Ossur Flex Run for leisure and fitness every day, had retained full joint articulation and suffered from no long-term pain or discomfort. They had a mass of $83 \mathrm{~kg}$ and as such used the correct stiffness category of foot according to the manufacturer's literature $[16,17]$. The selection of the participant and testing was conducted following Bournemouth University ethical approval (Reference ID: 4731).

The amputee was allowed 30 minutes to warm up with the ESR foot in the test environment (a 25 metre sports hall with wooden floor). The testing routine consisted of the sustained running of ten lengths of the hall $(250 \mathrm{~m}$ with nine turns) with the entire sequence logged at a frequency of $128 \mathrm{~Hz}$. The participant was allowed to choose his own pace and cadence with which he felt most comfortable and familiar. Both sagittal plane displacement and ground contact force were collected during the testing and averaged over 8 strides as shown in Table 1 . This table indicates the key parameters that describe the running. It is these six parameters (namely $2,5,7,8,9,10$ ) that should be replicated if the rig is to effectively match the amputee. Of these six parameters, three of them are purely a matter of using the same foot and adding mass to the carriage as to replicate the mass of the amputee. The remaining three are stance phase timing (or more appropriately in this instance 'ground contact time' as there is no stance phase of which to speak in the absence of a runner), maximum foot deflection and ground reaction force.

It is unrealistic to enter into this investigation assuming that all three of these variables can be matched. In reality only one can be purposefully replicated to that measured during amputee running. The remaining two variables will present themselves as a result of this first variable and the conditions of the foot and rig. For example if the rig were exercised with a progressively larger amplitude of the carriage until a ground reaction force of ' $x$ ' were achieved, the resulting ground contact time and maximum foot deflection could only be measured as a result of this ground reaction force. And the same is true if one of the other two variables were chosen as the driver. Of these three variables, the most practical to choose as the driver is maximum foot deflection. This can be easily viewed by the operator during testing without the need for additional instrumentation by simply marking one of the stanchions of the test rig.

TABLE I. RunNing CHARACTERISTICS OF THE AMPuteE ATHLETE DURING TESTING

\begin{tabular}{|c|c|c|}
\hline \multirow{2}{*}{$\begin{array}{c}\mathrm{Nr} \\
\text { Crt. }\end{array}$} & \multicolumn{2}{|c|}{ Amputee Characteristics } \\
\hline & Characteristics & Nominal Value \\
\hline 1 & Height of runner & $180 \mathrm{~cm}$ \\
\hline 2 & Weight of runner & $83 \mathrm{~kg}$ \\
\hline 3 & Cadence of runner & $1.08 \mathrm{~Hz}$ \\
\hline 4 & Time for a single stride & $728 \mathrm{~ms}$ \\
\hline 5 & Time for stance phase & $242 \mathrm{~ms}$ \\
\hline 6 & Time for swing phase & $486 \mathrm{~ms}$ \\
\hline 7 & Model of foot & Ossur Flex Run \\
\hline 8 & Category of foot & $6 \mathrm{Hi}$ \\
\hline 9 & Maximum displacement & $69.7 \mathrm{~mm}$ \\
\hline 10 & Ground reaction force & $1.70 \mathrm{kN}$ \\
\hline
\end{tabular}

\section{Ground Contact Point}

One additional variable to take into account before testing can commence is that of the contact point that the foot holds with the ground, which in fact varies throughout the stance phase of a single stride. While testing with the Ossur Category $6 \mathrm{Hi}[16,17]$ foot and a single amputee athlete the contact point was shown to travel from over $100 \mathrm{~mm}$ posterior of the front tip of metatarsal region of the foot (toe region) to the tip. However the rig is not capable of dynamically altering the contact point of the foot with the ground during the period of a single oscillation. Therefore a single ground contact point should be defined at which all testing must take place.

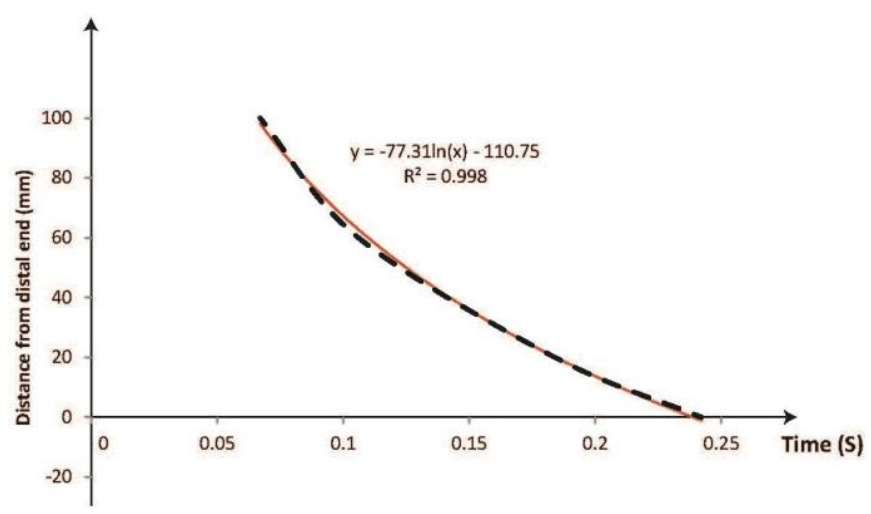

Fig. 2. (a) Curve demonstrating the shift in ground contact position relative to the distal end of the foot (toe region) (dashed line), (b) trend line (continuous line) [18]. 
The main purpose of this specific investigation is to establish if the amputee and the rig can be effectively compared for the basis of future work. This requires the maximum values to align, that is, the maximum foot deflection amplitude, maximum ground reaction force, maximum deflection timing, etc., and therefore the ground contact point chosen should be that which results at this same point of maximum foot deflection (\& displacement)/ ground force during normal running use.

During the testing with the Ossur Category $6 \mathrm{Hi}$ foot $[16$, 17] it was found that the timing of maximum deflection of the prosthetic foot during amputee running occurred (within measurable limits) exactly at the midpoint between heel strike and toe-off. As shown in Table 1 the total stance phase time was $242 \mathrm{~ms}$ meaning that maximum deflection must occur at $242 / 2=121 \mathrm{~ms}$. Using the graph in Fig. 2, it can be seen that at $121 \mathrm{~ms}$ after heel strike the ground contact point of the foot is $50 \mathrm{~mm}$ posterior of the tip of the foot. Therefore it was at this point that the toe-clamp portion of the rig was attached. This modification in ground contact point is also the reason for the change in maximum deflection. The testing showed the maximum deflection of the foot as $72 \mathrm{~mm}$ at a ground contact point of $40 \mathrm{~mm}$ from the toe. The ground contact point for the purpose of this test is being changed to $50 \mathrm{~mm}$ from the toe meaning that this overall deflection value of the foot is invalid. The single point characterisation testing was repeated but with a ground contact point of $50 \mathrm{~mm}$ rear of the toe. The resulting maximum deflection value was $69.7 \mathrm{~mm}$ and it is this value that is to be replicated by the rig.

\section{B. Method}

The rig carriage has a mass of $16.0 \mathrm{~kg}$ before any additional weights are added. Therefore to achieve a total mass of $83 \mathrm{~kg}$ a further $67 \mathrm{~kg}$ was required. A summary of the test conditions is shown in Table II. To run the test, the logger was started and the carriage exercised up and down using the input handle. Force was applied downwards on the input handle and it quickly became apparent at what moment in the oscillation cycle the force should be applied to ensure the most efficient build-up of energy in the prosthetic system.

TABLE II. TEST CONDITIONS

\begin{tabular}{|c|l|c|}
\hline $\begin{array}{c}\text { Nr } \\
\text { Crt. }\end{array}$ & \multicolumn{2}{|c|}{ Test Conditions } \\
\hline 1 & Test Name & Running Replication \\
\hline 2 & Foot on Test & Ossur Flex-Run Cat 6 Hi \\
\hline 3 & Carriage Mass & $16 \mathrm{~kg}$ \\
\hline 4 & Added Mass & $67 \mathrm{~kg}$ \\
\hline 5 & Total Mass & $83 \mathrm{~kg}$ \\
\hline 6 & Driving Variable & Foot displacement \\
\hline 7 & Variable Value & $69.7 \mathrm{~mm}$ \\
\hline
\end{tabular}

This coincided with the natural harmonic resonance of the system and is the subject of further discussion in this chapter.
Energy was applied in this timely manner until the target maximum foot deflection was achieved.

As discussed previously a mark was added to the righthand stanchion where the edge of the handle passes at 69.7 $\mathrm{mm}$ below the unloaded condition of the foot to act as a visual reference to the user. When the edge of the handle coincided with this mark the maximum deflection value had been reached. Having the required deflection been achieved it was found that the input force could be reduced in order to maintain the desired amplitude. This is demonstrated in Fig. 3 which is a trace of handle input force and foot deflection against time.

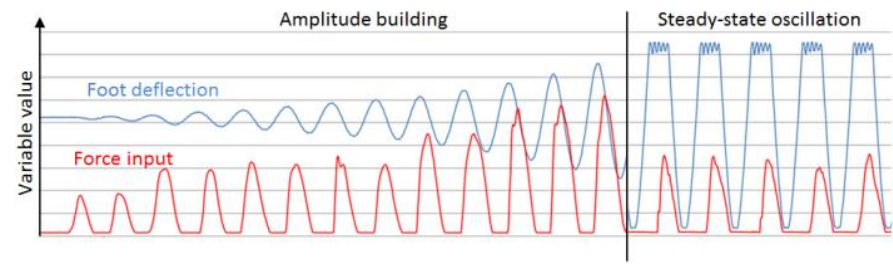

Fig. 3. Graph demonstrating the input force required to build-up the amplitude of oscillation of a prosthetic foot mounted on the test rig, compared with that required to maintain steady-state amplitude

\section{Results}

Fig. 4 shows the raw data that was collected for the 15 fulldisplacement oscillations. The $\mathrm{X}$-axis is the time code as generated by the data logger and the $\mathrm{Y}$-axis is displayed in volts as collected by the data logger.

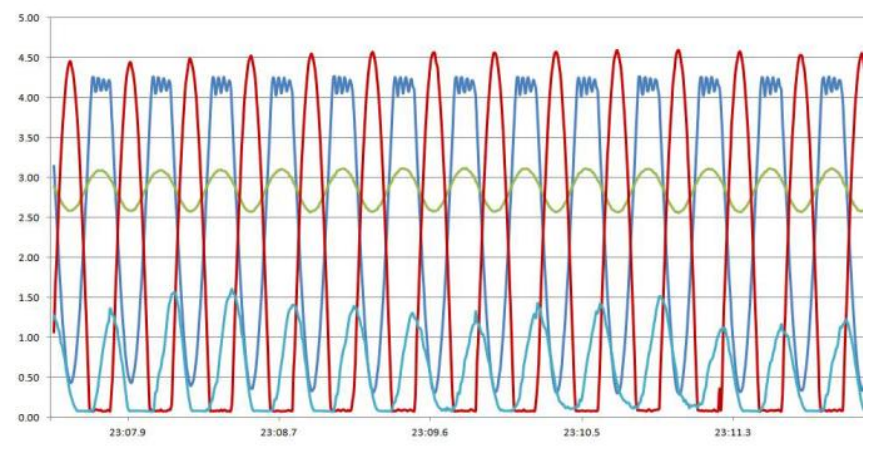

Fig. 4. Graph Raw data captured from all four logged channels with isolated oscillations

In order to understand the dynamic action of each of the variables a smaller sample of this trace is shown in Fig. 5 with some of the key points labelled. Shown are two full 'strides' (or complete oscillations) of the rig with all four channels overlaid. The $\mathrm{X}$-axis is still expressed as the time code generated by the data logger and the $\mathrm{Y}$-axis expressed in voltage as logged, and the important events of the stride are labelled.

Point $\mathrm{A}$ is the equivalent of heel strike in amputee running. This is the moment the foot first touches the ground plane and deflection begins. At this point the mass is moving in the $-\mathrm{Y}$ direction at its maximum velocity. At point $A$ it can be seen that the foot deflection and ground reaction force react simultaneously and mirror each other in an opposing apex. 


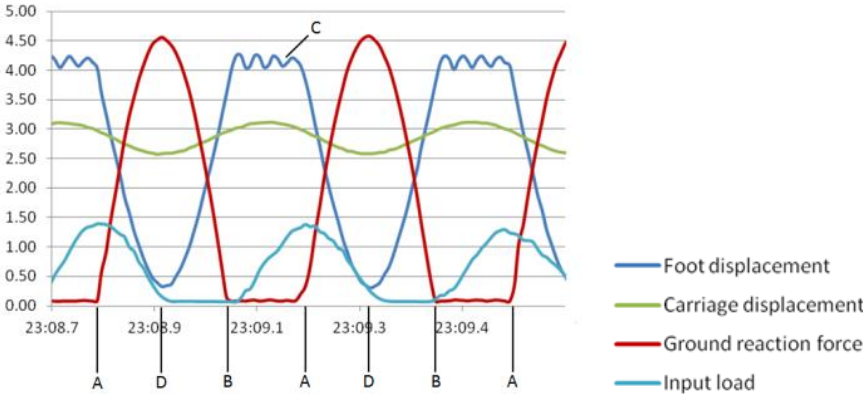

Fig. 5. Isolated oscillation cycles from figure 5.12 with key stride events labelled A, B, C, D.

The moment of maximum foot deflection (and maximum ground reaction force) is labelled as $\mathrm{D}$ at which point the velocity of the carriage is zero and the kinetic energy of point A has been converted into potential energy stored in the foot. Then the carriage changes direction and accelerates towards point $B$; the equivalent of toe-off in amputee running.

The foot leaves the ground plane and as it does so the toe of the foot, now unsupported by the rig, enters its own resonance. This harmonic is visible on the trace of foot deflection as measured by the vario-resistive sensor mounted to the foot and is labelled as point $\mathrm{C}$. Before the resonance has time to decay to zero the carriage returns towards the ground plane and once again the toe of the foot comes into contact with the ground reaction force load cell and is shown again as point $\mathrm{A}$.

Throughout this single oscillation the carriage displacement can be seen as a smooth sine wave and the trace of force input from the handle can also be seen. In order to generate more meaningful data from this information the time code was converted into milliseconds and each channel converted into a value of its native unit. Additionally the $\mathrm{X}$ and $\mathrm{Y}$ values of each of the measured variables was split and displayed on a separate graph. Only the centre ten oscillation cycles have been considered for data analysis to ensure the 'warm-up' (amplitude build-up) and 'warm-down' (amplitude decay) phases did not influence the data. The maximum value achieved by each variable for each oscillation during test was recorded and the mean calculated, as shown in Fig 6, Fig 7, Fig 8 and Fig 9.

Important to note is that due to the offset of the peaks of the various traces, the input force (Fig. 8) and the carriage displacement (Fig. 9) only feature 9 peaks instead of 10 . The mean peak of each of these traces was calculated and is tabulated in Table III where it is compared with the values obtained from the amputee athlete who was being mimicked.

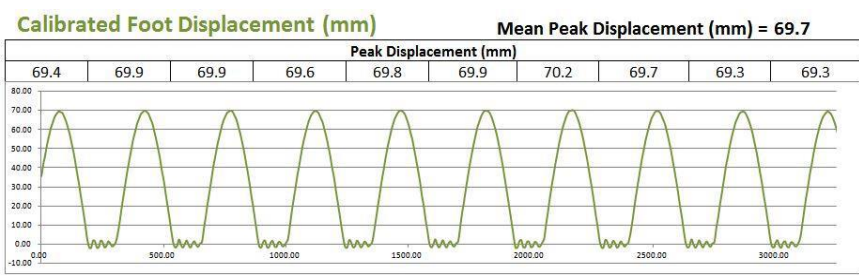

Fig. 6. Trace of foot deflection $(83 \mathrm{~kg})$

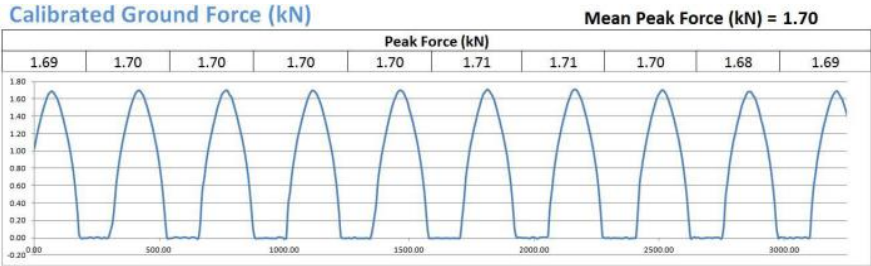

Fig. 7. Trace of ground reaction force $(83 \mathrm{~kg})$

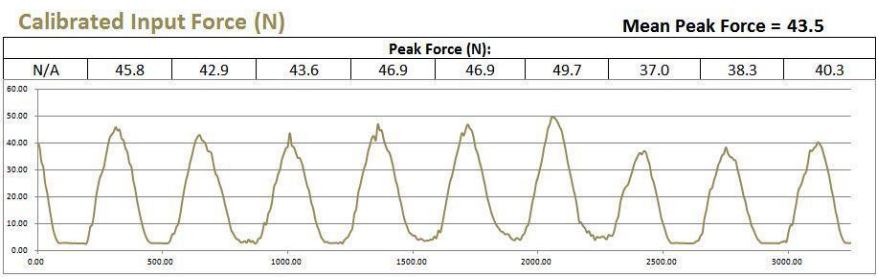

Fig. 8. Trace of input force $(83 \mathrm{~kg})$

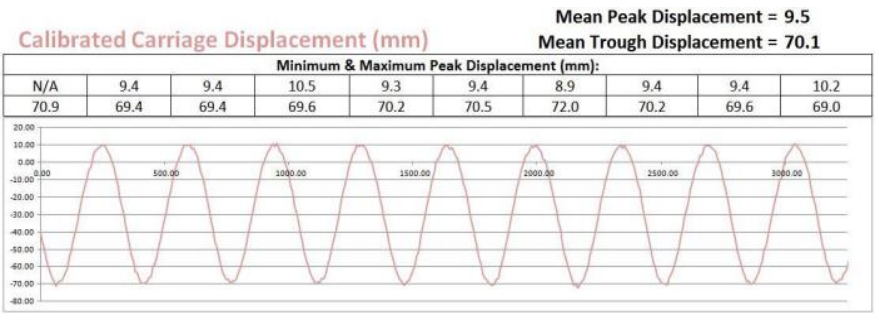

Fig. 9. Trace of carriage displacement $(83 \mathrm{~kg})$

As shown in Table III, if the maximum mean values are examined, within measureable limits the running action of the amputee subject is identical to the oscillation of the rig. For the purpose of this investigation the driving variable was foot deflection; this is the variable that was set out to be replicated from the recorded amputee running. As can be seen in Fig. 6 the intended value of $69.7 \mathrm{~mm}$ was not always perfectly achieved (as is inevitable with a human-operated system) but over the ten measured oscillations the mean value is ideal. The ground force is also identical which stands to reason. If the foot deflection is the same, then for this given deflection the force exerted must also be the same as previously measured. Above all, this result serves to confirm the repeatable setup of the foot deflection instrumentation and the accurate calibration of the ground force load cell.

It is logical to expect the recorded values of foot deflection and carriage displacement to align as it is the displacement of the carriage that is driving the deflection of the foot, but there is a discrepancy of $0.4 \mathrm{~mm}$. This could be a measurement or calibration error, but more likely it is a result of the misalignment between the medial mounting point of the foot to the base of the carriage and the centerline of the deflection transducer attached to the foot.

The setup of the deflection transducer requires the pivot of the rotary transducer to be directly below the centreline of the shank adapter used for attaching the foot to an amputee, but this is when the shank is perfectly vertical. When installed in the rig the shank is not vertical and indeed when the foot goes through deflection the shank portion of the foot rotates in the sagittal plane. The result of this is that the pivot of the rotary 
deflection transducer can never perfectly align with the centreline of the rig and as such will inevitably measure a small error when compared with the true vertical motion of the carriage. However it should be noted that for the purpose of this investigation it is the deflection of the foot that is more important as it is this variable that is being directly compared with what was measured during amputee testing.

TABLE III. AVERAgE DATA OVER 10 StRIDES For Rig VS. AMPUTEE RUNNING WITH IDENTICAL INPUT CONDITIONS (SD VALUE LISTED)

\begin{tabular}{|c|c|c|c|}
\hline \multirow{2}{*}{ Variable Name } & \multicolumn{3}{|c|}{ Table Column Head } \\
\cline { 2 - 4 } & $\begin{array}{c}\text { Rig } \\
\text { Measurement }\end{array}$ & SD & $\begin{array}{c}\text { Amputee } \\
\text { Measurement }\end{array}$ \\
\hline $\begin{array}{c}\text { Ground force (kN) } \\
\begin{array}{c}\text { Force Displacement } \\
(\mathrm{mm})\end{array}\end{array}$ & 1.70 & 0.01 & 1.70 \\
\hline $\begin{array}{c}\text { Minimum Carriage } \\
\text { Displacement (mm) }\end{array}$ & 9.5 & 0.28 & 69.7 \\
\hline $\begin{array}{c}\text { Maximum Carriage } \\
\text { Displacement (mm) }\end{array}$ & 70.1 & 0.89 & N/A \\
\hline $\begin{array}{c}\text { Input Force (N) } \\
\text { Stance Phase }\end{array}$ & 43.5 & 4.28 & Unknown \\
\hline $\begin{array}{c}\text { Timing/Ground } \\
\text { Contact Time (ms) }\end{array}$ & $235-245$ & 0 & $235-245$ \\
\hline
\end{tabular}

Equally important is the apparent ability of the rig to replicate the timing of the amputee. The ground contact time between the amputee and the rig is identical (to the nearest $10 \mathrm{~ms}$ ). At a logging frequency of $128 \mathrm{~Hz}$ it is impossible to differentiate between the two. This apparent replication of running style can be examined by comparing the foot deflection data for the entire stance-phase (Fig. 10). Data from ten strides (in the case of the amputee)/oscillations (in the case of the rig) is averaged in to a single set and the two resulting curves are overlaid.

Fig. 10 illustrates that whilst the overall ground contact time of the foot is identical between the rig and the amputee there are some notable differences in how the deflection of the foot progresses over the period of a single stride. Firstly when the foot first comes into contact with the ground the rate of deflection is greater during amputee running than it is on the rig (labelled A in Fig. 10). This is particularly apparent at the very early stages after heel strike. However at approximately $30 \mathrm{~mm}$ deflection this situation changes and it appears that the gradients of both traces become aligned. The data shows that the foot measured during amputee running reaches its point of maximum deflection fractionally before that from the rig (labelled B), but after the apex it can be seen that the opposite is true. The steeper gradient belongs to the data from the rig and as a result the two traces tend towards each other until they reach $0 \mathrm{~mm}$ deflection at the same time.

This phenomenon is a result of the ground contact point changing along the metatarsal region of the foot. However the rig set-up uses a single effective assumed ground contact point. As such, when the amputee first lands the portion of the foot that touches the ground first is posterior to that point used on the rig. This results in a decreased lever-arm ratio of the foot which equates to a higher relative stiffness and an increased rate of deflection. As the ground contact point progresses forward towards the anterior edge of the foot the reverse is true. The effective stiffness of the foot becomes progressively less and the subsequent rate of deflection lower. The moving ground contact point exhibiting itself in this way and the fact that both feet exhibit the same ground contact time adds validity to the assumed ground contact point used on the rig.

The second notable difference is the resonance (labelled $\mathrm{C}$ in Fig. 10) that occurs after toe-off (when the foot leaves the ground). Both methods of testing exhibit resonance at this stage of the stride but the nature of this oscillation is different. During amputee testing it was apparent that the toe underwent one or two progressively decaying oscillations. During rig testing however this changed to four distinct and marginally decaying oscillations at significantly greater amplitude. The oscillations are always arrested by the foot coming into contact with the ground plane once more. This harmonic disparity is a result of the different setup of the two investigations. During amputee testing every effort was made not to add mass to the distal end of the foot so not to influence the running action of the individual. Added mass at the distal end of the foot might have affected the swing phase of the stride as the amputee works to reposition his foot. However during rig testing the mounting of the foot was a critical concern and hardware was added to the metatarsal region in order to clamp the foot. This hardware included the two bearings that allowed the rotational degree of freedom in the sagittal plane as required by the mounting strategy that was used.

The third difference is that of the duration of 'zero deflection' following toe-off. This is represented by the length of each of the traces. However this is easily explained as what is probably the greatest difference between testing using an amputee and on the rig. The traces shown in Fig. 10 illustrate the duration of a single stride or oscillation. On the rig the foot returns to the ground plane after approximately $330 \mathrm{~ms}$ to begin another cycle. However the foot of the amputee does not come into contact with the ground until approximately $720 \mathrm{~ms}$ because the process is interrupted by the action of the contralateral leg. The foot of the amputee is in the air for a significantly longer time whilst the other leg is undergoing the stance phase of the stride.

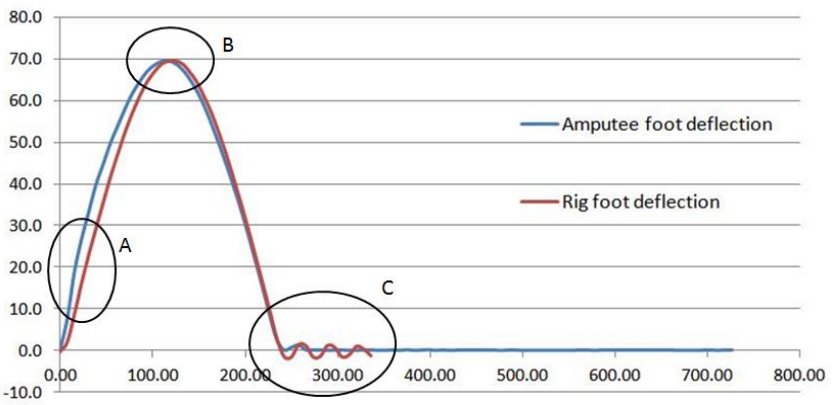

Fig. 10. Traces of foot deflection $(83 \mathrm{~kg})$ for both the rig and amputee testing phases

\section{CONCLUSIONS}

The objective of this study was to assess the design of a rig that was able to mimic amputee running. This allows individual 
variables to be modified to improve the understanding of amputee running by recording and manipulating some of the variables that contribute to the response of the foot and in turn enabling optimisation of the parameters to improve the prosthetic foot's performance. The validation was based on the assumptions that:

- the mass of the runner is equivalent to that of the carriage (with masses attached)

- $\quad$ the same (or an identical) foot is used

- $\quad$ the ground reaction force OR foot deflection is copied

- the ground contact point selected to represent the running action is equivalent to that achieved at the point of maximum foot deflection during amputee running (in this case $50 \mathrm{~mm}$ rear of the toe tip)

If these four conditions are satisfied the rig can simulate the running action of an amputee runner and return similar ground contact time. This establishes a known and understood link between the rig and amputee. It has also been shown that if a small input force is applied in a timed and sympathetic manner to the prosthetic spring-mass system the amplitude of oscillation (and therefore energy stored in the system) increases. This agrees with the views of $[12,13,14,15,19$, 20]. This baseline of energy input is required to maintain the amplitude of oscillation. If greater amplitude is to be achieved, a greater force input is required (in the same timely manner). If the input force is removed the amplitude of oscillation will decay to zero according to the frictional losses in the system.

Furthermore the ground contact point ( $50 \mathrm{~mm}$ rear of the tip of the toe) could change depending on running velocity, foot deflection, stride length, stride frequency or even the individual set up of the prosthetic foot. Previously it was shown that the change in ground contact point (and therefore the boundary conditions) has a dramatic effect on the effective stiffness of the foot. Additional investigations regarding rig design and design parameters should address this in order to understand both the magnitude of change of the ground contact area for different styles of running (across a range of speeds, stride lengths, etc.) and how relevant this change would be, for different type of prosthetic foots.

\section{ACKNOWLEDGMENT}

The author has received exceptional support from two specific organizations; Ossur UK for their assistance in understanding their specific prosthetic devices and the supply of a multitude of feet and foot coverings to allow repeatable test work to be conducted, and MSR Electronics GmbH for the supply of their flagship data logger. Thanks must also go to Dr Jan Walter Schroeder and Richard Hawkins Esq. for the design of the electronic circuits used in the various signal amplifiers and conditioner case as detailed in this report.

\section{REFERENCES}

[1] S. Au, M. Berniker, and H. Herr, "Powered ankle-foot prosthesis to assist levelground and stair-descent gaits", Neural Networks. 21, pp.654$666,2008$.

[2] A. Hansen, D.S. Childress, S.C. Miff, S.A. Gard, and K.P. Mesplay, "The human ankle during walking: implications for the design of biomimetic ankle prostheses", Journal of Biomechanics. 37, pp.14671474,2004

[3] J.W. Michael, R.S. Gailey, and J.H. Bowker, "New Developments in Recreational Prostheses and Adaptive Devices for the Amputee", Clinical Orthopaedics \& Related Research.Section I, 1990.

[4] R. Versluys, P. Beyl, M. Van Damme, A. Desomer, R. Van Ham, and D. Lefeber, "Prosthetic feet: State-of-the-art review and the importance of mimicking human anklefoot biomechanics", Disability and Rehabilitation: Assistive Technology. 4(2), pp. 65-75, 2009.

[5] C.P. McGowan, A.M. Grabowski, W.J. McDermott, H.M. Herr, and R. Kram, "Leg stiffness of sprinters using running-specific prostheses. Journal of the Royal Society Interface”, 9, pp. 1975-1982, 2012.

[6] R. Blickhan, and R. J. Full, "Similarity in multilegged locomotion: bouncing like a monopode", Journal of Comparative Physiology. A 173, pp. 509-517, 1993.

[7] R. Alexander, "A model of bipedal locomotion on compliant legs", Philosophical Transactions of the Royal Society of London. B 338, pp. 189-198, 1992.

[8] M. Geil, "Energy Loss and Stiffness Properties of Dynamic Elastic Response Prosthetic Feet", Prosthetic and Orthotic Science. 13(3), pp. 70-73, 2001.

[9] J. Buckley, "Biomechanical adaptations of transtibial amputee sprinting in athletes using dedicated prostheses", Clinical Biomechanics. 15(5), pp.352-8, 2000 .

[10] J.M. Czerniecki, A. Gitter, C.J. Munro, "Joint moment and muscle power output characteristics of below knee amputees during running: the influence of energy storing prosthetic feet", Biomechanics, 24(3-4), pp.271-2, 1991 .

[11] G-P. Bruggemann, A. Arampatzis, F. Emrich, W. Potthast, "Biomechanics of double transtibial amputee sprinting using dedicated sprinting prostheses" Sports Technology. No. 4-5, pp.220-227, 2008.

[12] J. Lehmann, R. Price, S. Boswell-Bessette, A. Dralle, and K. Questad, "Comprehensive Analysis of Dynamic Elastic Response Feet: Seattle Ankle/Lite Foot Versus SACH Foot", Archives of Physical Medicine \& Rehabilitation. 74(8), pp. 853-861, 1993.

[13] J. Lehmann, R. Price, S. Boswell-Bessette, A. Dralle, K. Questad, and B.J. deLateur, "Comprehensive Analysis of Energy Storing Prosthetic Feet: Flex Foot and Seattle Foot Versus Standard SACH Foot. Archives of Physical Medicine \& Rehabilitation”, 74(11), pp. 1225-1231, 1993.

[14] S. Noroozi, P. Sewell, A.G.A. Rahman, J. Vinney, O.Z. Chao, and B. Dyer, "Performance enhancement of bi-lateral lower-limb amputees in the latter phases of running events: an initial investigation", Journal of Sports Engineering and Technology. 227(2), pp.105-115, 2012.

[15] S. Noroozi, P. Sewell, A.G.A. Rahman, J. Vinney, O.Z. Chao, and B. Dyer, "Modal Analysis of Composite Prosthetic Energy-Storing-andReturning Feet: An Initial Investigation", Journal of Sports Engineering and Technology. 227(1), pp.39-48, 2012.

[16] Ossur products catalogue, P161. www.ossur.co.uk (Accessed 16th January 2015).

[17] Ossur Instructions for 2012. http://assets.ossur.com/library/25848/FlexRun\%20with\%20Nike\%20Sole_0153_IFU.pdf (Accessed 16th January 2015).

[18] J. Hawkins, S. Noroozi, M. Dupac, and P. Sewell, "Development of a wearable sensor system for dynamically mapping the behavior of an energy storing and returning prosthetic foot: A proof of concept", Measurement Science Review. 16(3), pp. 174-182, 2016.

[19] K. Lechler, and M. Lilja, "Lower extremity leg amputation: an advantage in running?", Sports Technology. 1(4-5), pp. 229-234, 2008 ..

[20] K. Lechler, "Lower-Limb Prosthetics - Design Improvements of a Prosthetic Spring Foot”, American Journal of Physics. 36, pp. 4-7, 2005. 